\title{
SOLVING NONLINEAR THERMAL PROBLEMS OF FRICTION BY USING METHOD OF LINES
}

\author{
Ewa $\mathrm{OCH}^{*}$ \\ ${ }^{*}$ Faculty of Mechanical Engineering, Bialystok University of Technology, 45C Wiejska Street, Bialystok, 15-351, Poland \\ e.och@doktoranci.pb.edu.pl
}

\begin{abstract}
One-dimensional heat conduction problem of friction for two bodies (half spaces) made of thermosensitive materials was considered. Solution to the nonlinear boundary-value heat conduction problem was obtained in three stages. At the first stage a partial linearization of the problem was performed by using Kirchhoff transform. Next, the obtained boundary-values problem by using the method of lines was brought to a system of nonlinear ordinary differential equations, relatively to Kirchhoff's function values in the nodes of the grid on the spatial variable, where time is an independent variable. At the third stage, by using the Adams's method from DIFSUB package, a numerical solution was found to the above-mentioned differential equations. A comparative analysis was conducted (Och, 2014) using the results obtained with the proposed method and the method of successive approximations.
\end{abstract}

Key words: Method Of Lines, Thermal Problems Of Friction, Thermosensitive Materials

\section{INTRODUCTION}

Almost all elements of machine that work together are accompanied by the friction processes. Influenced by the friction forces, on the contact surfaces of the body, the heat is generated, which as the heat fluxes - penetrates the elements of the system and heats them up. The temperature increase may lead to changes in physical and chemical properties of friction materials, friction coefficients and termomechanical wear, etc. (Chichinadze et al., 1979; Rhee et al., 1991; Kalin, 2004).

One of the methods to estimate the distribution of transient temperature fields in elements of heavily loaded nodes of friction is the use of analytical models, where the real friction elements (pad, disk) are replaced by half-limited (half space) or limited (layer) bodies (Nosko et al., 2009; Kuciej, 2012; Yevtushenko and Kuciej, 2012). Most of the models were developed basing on the solution to linear boundary-values problems of heat conduction, where changes in sliding velocity and pressure with time; inhomogeneity of friction materials; or different types of boundary conditions on the friction surface and on the free surfaces of elements were all taken into account (Sazonov, 2008; Belyakov and Nosko, 2010; Yevtushenko et al., 2013). But at the same time, the above solutions do not take into account the changes (caused by the increase of temperature) in coefficients of friction and wear, and thermal properties of materials.

Dependency of coefficients of friction and wear on the temperature were considered when the one-dimensional nonlinear frictional heating models were developed in articles (Olesiak et al., 1997; Evtushenko and Pir'ev, 1999) and monographs (Pyr'yev, 2004; Awrejcewicz and Pyr'yev, 2009).

Models that take into account the change of the thermal-physical properties of friction materials along with increasing temperature were proposed for the materials with a so-called simple thermal nonlinearity in articles (Och, 2013, Yevtushenko et al., 2014a, b), and for the materials with an arbitrary nonlinearity - in articles (Och, 2014; Yevtushenko et al., 2014c, 2015). Solutions to the respective thermal problems of friction were obtained by using the iterative methods (Kushnir, Popowych, 2011).

The main purpose of this paper is to show the effectiveness of the methods of lines in solving nonlinear boundary-value problems of heat conduction, with the heat generation due to friction taken into account.

\section{STATEMENT OF THE PROBLEM}

Let two thermally sensitive half spaces be pressed at infinity by constant pressure $p_{0}$ along the axis $z$ (Fig. 1). At the time $l=0$, taken as the initial, bodies begin to slide relatively to each other at a constant speed $V_{0}$ in the direction of the $y$ axis in accordance with a Cartesian coordinate system $O x y z$. The initial temperature of the bodies is the same and equal to $T_{0}$. On the contact surface, under the influence of friction forces, the heat is generated, which in the form of heat fluxes penetrates each bodies in such a way that their sum is equal to the specific power of friction $q_{0}=f V_{0} p_{0}$ (Yevtushenko and Kuciej, 2012). Thermal contact of considered bodies is imperfect, i.e. through the friction surface the heat flow takes place at a constant value of the thermal conductivity of contact coefficient. We assume the Podstrigach's condition of imperfect thermal contact, which take into account the thermal resistance of a thin layer between bodies at friction (Podstrigach, 1963). Reviews of researches into imperfect frictional thermal contact are given in the papers (Nosko et al., 2009, Belyakov and Nosko, 2010; Kuciej, 2012).

Further, all values referring to the upper and lower half spaces will respectively have subscripts 1 and 2 .

We assume that the coefficients of thermal conductivity $K_{l}$ and heat capacity $c_{l}$ of the bodies material depend on the temperature $K_{l}, l=1,2$ :

$K_{l}\left(T_{l}\right)=K_{l, 0} K_{l}^{*}\left(T_{l}\right), K_{l, 0} \equiv K_{l}\left(T_{0}\right)$,
$c_{l}\left(T_{l}\right)=c_{l, 0} c_{l}^{*}(T), c_{l, 0} \equiv c_{l}\left(T_{0}\right)$,

and their density $\rho_{l}, l=1,2$ is constant. 
$\left.\frac{\partial \Theta_{2}}{\partial \zeta}\right|_{\zeta=0}-\left.K_{0}^{*} \frac{\partial \Theta_{1}}{\partial \zeta}\right|_{\zeta=0}=1, \tau>0$
$\left.\frac{\partial \Theta_{2}}{\partial \zeta}\right|_{\zeta=0}+\left.K_{0}^{*} \frac{\partial \Theta_{1}}{\partial \zeta}\right|_{\zeta=0}=B i\left[T_{1}^{*}(0, \tau)-T_{2}^{*}(0, \tau)\right], \tau>0$

$\Theta_{l}^{(i)}(\zeta, \tau) \rightarrow 0, \quad|\zeta| \rightarrow \infty, \quad \tau \geq 0, \quad l=1,2$

$\Theta_{l}^{(i)}(\zeta, 0)=0, \quad|\zeta|<\infty, \quad l=1,2$

where:

$k_{l}^{*}\left(T^{*}\right)=K_{l}^{*}\left(T^{*}\right) / c_{l}^{*}\left(T^{*}\right), l=1,2$

Solution to partially linearized boundary-values problem (12)-(17) we obtain by using the methods of lines (Hall, Watt, 1976).

\section{METHOD OF LINES}

$\frac{\partial^{2} T_{1}^{*}}{\partial \zeta^{2}}=\frac{1}{k_{0}^{*} k_{1}^{*}\left(T_{1}^{*}\right)} \frac{\partial T_{1}^{*}}{\partial \tau}, \zeta>0, \tau>0$

$\frac{\partial^{2} T_{2}^{*}}{\partial \zeta^{2}}=\frac{1}{k_{2}^{*}\left(T_{2}^{*}\right)} \frac{\partial T_{2}^{*}}{\partial \tau}, \zeta<0, \tau>0$

$\left.K_{2}^{*}\left(T_{2}^{*}\right) \frac{\partial T_{2}^{*}}{\partial \zeta}\right|_{\zeta=0^{-}}-\left.K_{0}^{*} K_{1}^{*}\left(T_{1}^{*}\right) \frac{\partial T_{1}^{*}}{\partial \zeta}\right|_{\zeta=0^{+}}=1, \quad \tau>0$

$\left.K_{2}^{*}\left(T_{2}^{*}\right) \frac{\partial T_{2}^{*}}{\partial \zeta}\right|_{\zeta=0^{-}}+\left.K_{0}^{*} K_{1}^{*}\left(T_{1}^{*}\right) \frac{\partial T_{1}^{*}}{\partial \zeta}\right|_{\zeta=0^{+}}=$

$=B i\left[T_{1}^{*}\left(0^{+}, \tau\right)-T_{2}^{*}\left(0^{-}, \tau\right)\right], \tau>0$

$T_{l}^{*}(\zeta, \tau) \rightarrow T_{0}^{*}, \quad|\zeta| \rightarrow \infty, \quad l=1,2$

$T_{l}(\zeta, 0)=T_{0}^{*}, \quad|\zeta|<\infty, l=1,2$

$\zeta=\frac{z}{a}, \quad \tau=\frac{k_{2,0} t}{a^{2}}, \quad K_{0}^{*}=\frac{K_{1,0}}{K_{2,0}}, \quad k_{0}^{*}=K_{0}^{*} \frac{\rho_{2} c_{2,0}}{\rho_{1} c_{1,0}}$

$B i=\frac{h a}{K_{2,0}}, T_{a}=\frac{q_{0} a}{K_{2,0}}, T_{0}^{*}=\frac{T_{0}}{T_{a}}, T_{l}^{*}=\frac{T_{l}}{T_{a}}$

$k_{l}^{*}\left(T_{l}^{*}\right)=\frac{K_{l}^{*}\left(T_{l}^{*}\right)}{c_{l}^{*}\left(T_{l}^{*}\right)}, l=1,2$

where: $\alpha$ - is the effective depth of the heat penetration (Chichinadze et al., 1979).

\section{KIRCHHOFF TRANSFORMATION} 1894):

We introduce the Kirchhoff's functions $\Theta_{l}(\zeta, \tau)$ (Kirchhoff,

$\Theta_{l}(\zeta, \tau)=\int_{T_{0}^{*}}^{T^{*}} K_{l}^{*}\left(T_{l}^{*}\right) d T_{l}^{*}, \quad l=1,2$

As a result, we obtained a partially linearized boundary-value problems in relation to the functions $\Theta_{l}(\zeta, \tau)$ :

$\frac{\partial^{2} \Theta_{1}}{\partial \zeta^{2}}=\frac{1}{k_{0}^{*} k_{1}^{*}\left(T_{1}^{*}\right)} \frac{\partial \Theta_{1}}{\partial \tau}, \zeta>0, \tau>0$

$\frac{\partial^{2} \Theta_{2}}{\partial \zeta^{2}}=\frac{1}{k_{2}^{*}\left(T_{2}^{*}\right)} \frac{\partial \Theta_{2}}{\partial \tau}, \zeta<0, \tau>0$

We choose layer in each half spaces $0 \leq|\zeta| \leq \delta_{l}, l=1,2$ in such a way that $|\zeta|=\delta_{l}$ boundary condition can be fulfilled (16). Let us divide compartments $\left[0, \delta_{l}\right], l=1,2$ into $n_{l} \in N$ parts with the points:

$\zeta_{l, j}=(-1)^{l+1} j \Delta \zeta_{l}, \Delta \zeta_{l}=\delta_{l} / n_{l}, j=0,1, \ldots, n_{l}, l=1,2$

On the grid (19) we introduce central finite-difference approximations of partial derivatives (Ozisik, 2000):

$\frac{\partial \Theta_{l}(\zeta, \tau)}{\partial \zeta} \approx \frac{\Theta_{l, j+1}(\tau)-\Theta_{l, j-1}(\tau)}{2 \Delta \zeta_{l}}$

$\frac{\partial^{2} \theta_{l}(\zeta, \tau)}{\partial \zeta^{2}} \approx \frac{\Theta_{l, j+1}(\tau)-2 \theta_{l, j}(\tau)+\Theta_{l, j-1}(\tau)}{\left(\Delta \zeta_{l}\right)^{2}}$

where: $\Theta_{l, j}(\tau) \equiv \Theta_{l, j}\left(\zeta_{l, j} \tau\right), \tau \geq 0, j=0,1, \ldots, n_{l}, l=1,2$.

Taking into account the formulas (19)-(21), the boundary-value problem (12)-(17) can be written in the form:

$$
\begin{aligned}
& \frac{\Theta_{1, j+1}(\tau)-2 \Theta_{1, j}(\tau)+\Theta_{1, j-1}(\tau)}{\left(\Delta \zeta_{1}\right)^{2}}=\frac{1}{k_{0}^{*} k_{1, j}^{*}(\tau)} \frac{d \Theta_{1, j}(\tau)}{d \tau}, \\
& j=0,1, \ldots, n_{1}, \\
& \frac{\Theta_{2, j+1}(\tau)-2 \Theta_{2, j}(\tau)+\Theta_{2, j-1}(\tau)}{\left(\Delta \zeta_{2}\right)^{2}}=\frac{1}{k_{2, j}^{*}(\tau)} \frac{d \Theta_{2, j}(\tau)}{d \tau}, \\
& j=0,1, \ldots, n_{2}, \\
& \frac{\Theta_{2,1}(\tau)-\Theta_{2,-1}(\tau)}{2 \Delta \zeta_{2}}-K_{0}^{*} \frac{\Theta_{1,1}(\tau)-\Theta_{1,-1}(\tau)}{2 \Delta \zeta_{1}}=1 \\
& \frac{\Theta_{2,1}(\tau)-\Theta_{2,-1}(\tau)}{2 \Delta \zeta_{2}}+K_{0}^{*} \frac{\Theta_{1,1}(\tau)-\Theta_{1,-1}(\tau)}{2 \Delta \zeta_{1}} \\
& =B i\left[T_{1,0}^{*}(\tau)-T_{2,0}^{*}(\tau)\right]
\end{aligned}
$$

$\Theta_{l, n_{l}+1}(\tau)-\Theta_{l, n_{l}-1}(\tau)=0, l=1,2$

$\Theta_{l, j}(0)=0, j=0,1, \ldots, n_{l}, l=1,2$

where:

$T_{l, j}^{*}(\tau) \equiv T^{*}\left(\zeta_{l, j}, \tau\right), k_{l, j}^{*}(\tau) \equiv k_{l}^{*}\left[T_{l, j}^{*}(\tau)\right]$

$\tau>0, j=0,1, \ldots, n_{l}$

From the boundary conditions (24) and (25) on the contact surface $\zeta=0$ we find: 


$$
\begin{aligned}
& \Theta_{l,-1}(\tau)=\Theta_{l, 1}(\tau)+(-1)^{l-1} g_{l}(\tau), \tau \geq 0, l=1,2 \\
& g_{1}(\tau)=\left\{1-B i\left[T_{1,0}^{*}(\tau)-T_{2,0}^{*}(\tau)\right]\right\} \Delta \zeta_{1} / K_{0}^{*} \\
& g_{2}(\tau)=\left\{1+B i\left[T_{1,0}^{*}(\tau)-T_{2,0}^{*}(\tau)\right]\right\} \Delta \zeta_{2}
\end{aligned}
$$

By introducing functions:

$$
A_{1, j}(\tau)=\frac{k_{0}^{*} k_{1, j}^{*}(\tau)}{\left(\Delta \zeta_{1}\right)^{2}}, A_{2, j}(\tau)=\frac{k_{2, j}^{*}(\tau)}{\left(\Delta \zeta_{2}\right)^{2}}
$$

and taking into account the relations (26), (29)-(31), Cauchy problem for a system of ordinary differential equations (22)-(27) we write in the form:

$$
\begin{aligned}
& \frac{d \Theta_{l, 0}(\tau)}{d \tau}=A_{l, 0}(\tau)\left[2 \Theta_{1,1}(\tau)-2 \Theta_{1,0}(\tau)+(-1)^{l-1} g_{l}(\tau)\right] \\
& \tau>0 \\
& \frac{d \Theta_{l, j}(\tau)}{d \tau}=A_{l, j}(\tau)\left[\Theta_{l, j+1}(\tau)-2 \Theta_{l, j}(\tau)+\Theta_{l, j-1}(\tau)\right] \\
& \tau>0, j=1,2, \ldots, n_{l}-1 \\
& \frac{d \Theta_{l, n_{l}}(\tau)}{d \tau}=A_{l, n_{l}}(\tau)\left[2 \Theta_{l, n_{l}-1}(\tau)-2 \Theta_{l, n_{l}}(\tau)\right], \tau>0 \\
& \Theta_{l, j}(0)=0, j=0,1, \ldots, n=n_{1}+n_{2}+2, l=1,2
\end{aligned}
$$

Integration of the problem (33)-(36) is carried out by using the Adams's method. The method was carried out in DIFSUB procedure written in FORTRAN (Gear, 1971). This procedure is used to perform one step of the independent variable $\tau$, and therefore the numerical integration of the initial value problem (33)(36) at a predetermined time interval requires a multiple "call" of this procedure. The detailed information about the package DIFSUB are contained in the monograph (Krupowicz, 1986).

As a result of solving the initial problem (33)-(36), the values $\Theta_{l, j}(\tau)$ of Kirchhoff's function (11) were found in the nodes of the grid (19) at a specific point of dimensionless time $\tau$. In order to make the transition from Kirchhoff;s function to respective values $T_{l, j}^{*}(\tau)$ of dimensionless temperature, we must define the functions $K_{l}^{*}\left(T_{l}^{*}\right)$ and $c_{l}^{*}\left(T_{l}^{*}\right), l=1,2$ in the formulas (1). We assume they have the polynomial form:

$K_{l}^{*}\left(T_{l}^{*}\right)=\sum_{n=0}^{N_{l}} a_{l, n}\left(T_{l}^{*}\right)^{n}, c_{l}^{*}\left(T^{*}\right)=\sum_{n=0}^{M_{l}} b_{l, n}\left(T_{l}^{*}\right)^{n}$ $l=1,2$

with known coefficients $a_{l, n}$ and $b_{l, n}$ (Yune, Bryant 1989). Then, with regard to equations (11) and (37), the relationship between the dimensionless temperature and the Kirchhoff's function will be also polynomial (Och, 2014):

$T_{l, j}^{*}(\tau)=\sum_{n=0}^{N_{l}} c_{l, n}\left[\Theta_{l, j}(\tau)\right]^{n}, \tau \geq 0, j=0,1, \ldots, n_{l}$

$l=1,2$.

We also note that at the constant thermal properties of the materials $\left(K_{l}^{*}\left(T^{*}\right)=c_{l}^{*}\left(T^{*}\right)=1\right)$ from the formula (11) it follows the linear relationship between temperature and the Kirchhoff function:

$T_{l}^{*}(\zeta, \tau)=T_{0}^{*}+\Theta_{l}^{*}(\zeta, \tau),|\zeta|<\infty, l=1,2$

\section{NUMERICAL ANALYSIS}

Calculations have been performed for the same materials of the friction pair (aluminium AL MMC - metal-cermic FMC-845) and at the same input parameters as in article (Och, 2014), in which analytical-numerical solution of boundary-values problem (2)-(7) was found by method of successive approximations. Thermophysical properties of materials at initial temperature $T_{0}=20^{\circ} \mathrm{C}$ are given in Tab. 1 , and the values of the coefficients $\left(a_{l, n}, b_{l, n}, c_{l, n}\right)$ in the formulas (37)-(39) - in Tab. 2, in the above-mentioned paper. Dimensionless thickness of the layers were the same and equal $\delta_{1}=\delta_{2}=\delta_{0}=5$, the numbers of compartments breakdown of each layer were the same, too: $n_{1}=n_{2}=n_{0}$. Thus, the initial problem (33)-(36) with a number of equations $n=2 n_{0}+2$ was solved by the procedure DIFSUB.

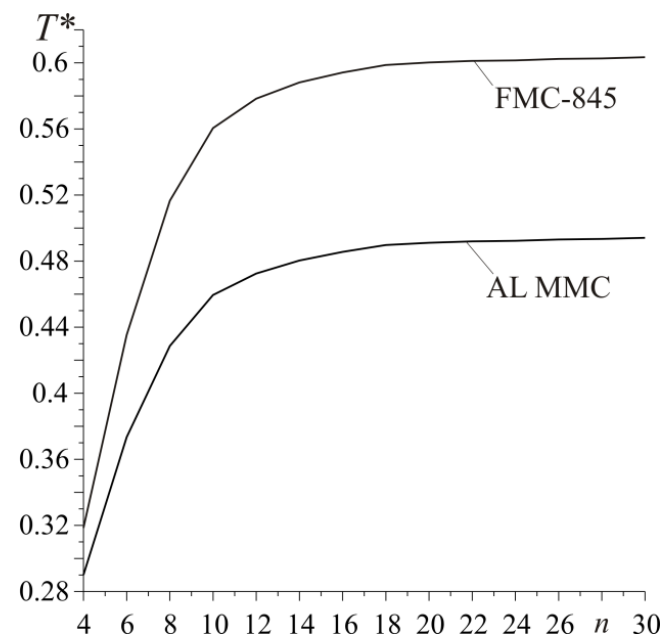

Fig. 2. Dependence of dimensionless temperature $T_{l}^{*}, l=1,2$ on the contact surface $\zeta=0$ on parameter $n$, at $\tau=2$

The convergence of the calculation process of dimensionless temperature on the friction surface depending on the parameter $\mathrm{n}$ inversely related to the dimensionless length of the step grid $\Delta \zeta_{1}=\Delta \zeta_{2}=\delta_{0} / n_{0}$, is shown in Fig. 2. For a given relative accuracy of the calculations EPS $=10^{-6}$, the temperatures of both materials are almost unchanged at $n \geq 20$. The thermal conductivity of metal-ceramic FMC-845 is less than the conductivity of the aluminum matrix composite AL MMC (Och, 2014). As the result, the temperature of the friction surface of the metal-ceramics is higher than the temperature on the surface of the composite.

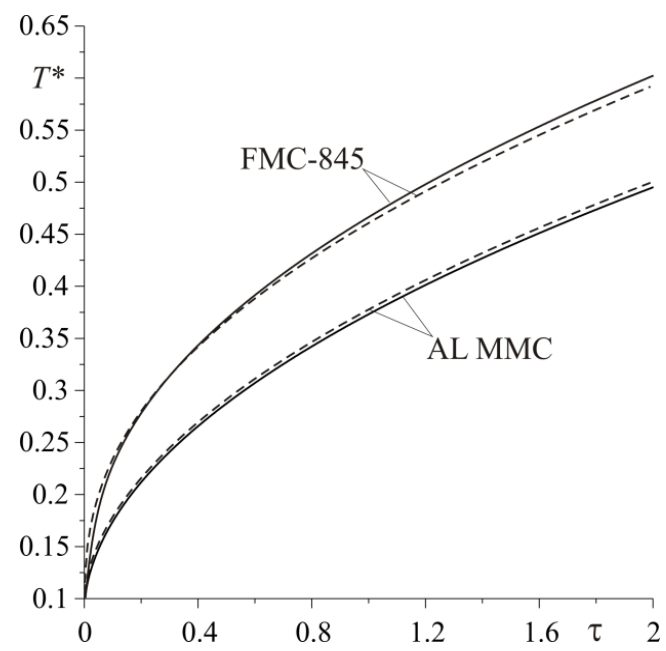

Fig. 3. Evolutions of dimensionless temperature $T_{l}^{*}, l=1,2$ on the contact surface $\zeta=0$, obtained by using the method of lines (solid lines), and by using the method of successive approximations (Och, 2014, dashed lines). 
Evolutions of dimensionless temperature on the contact surface during sliding at a constant speed for the considered friction pair, are presented in Fig. 3. In this figure solid lines shows the results of calculations obtained by method of lines, using the procedure DIFFSUB, while the dashed lines presents the results of calculations obtained by successive approximations and presented in the article (Och, 2014). We may see that the dimensionless temperature curves calculated for aluminum matrix composite AL MMC by using both methods are practically the same. For metal-ceramic FMC-845 from about half of the heating time the slight difference between the respective curves is noticeable.

Isotherm of friction elements AL MMC and FMC-845 in the coordinate system $\zeta \tau$ are presented in Fig. 4 . With the beginning of sliding temperature in any cross-section relative to the $\zeta$ increases. Maximum temperatures are reached at the surfaces of the friction elements at $\zeta=0$ and the temperature of both bodies decreases with increasing distance from this surface. High thermal conductivity of $\mathrm{AL} \mathrm{MMC}$ and substantially smaller of FMC-845 causes that the effective depth of heat penetration of aluminium composite is two times greater than the metal-ceramic.

When using iterative methods to solve nonlinear thermal problems of friction at the initial "zero" step, we must have a solution (preferably the analytical one) to the corresponding linear problems (Yevtushenko et al., 2015). The methodology proposed in this paper proves to be effective when it is difficult or even impossible to obtain such analytical solutions of linear problems. For example there is a change of the specific power of friction in time in the form $q(\tau)=q_{0} q^{*}(\tau)$, when $q^{*}(\tau)=1+\sin (\omega \tau), \tau \geq 0$, where for $\omega=0$ we obtain the problem considered above. Change with time of friction elements temperature at constant $(\omega=0)$ and oscillating $(\omega=50)$ (Kuciej, 2011) specific power of friction is presented in Fig. 5.

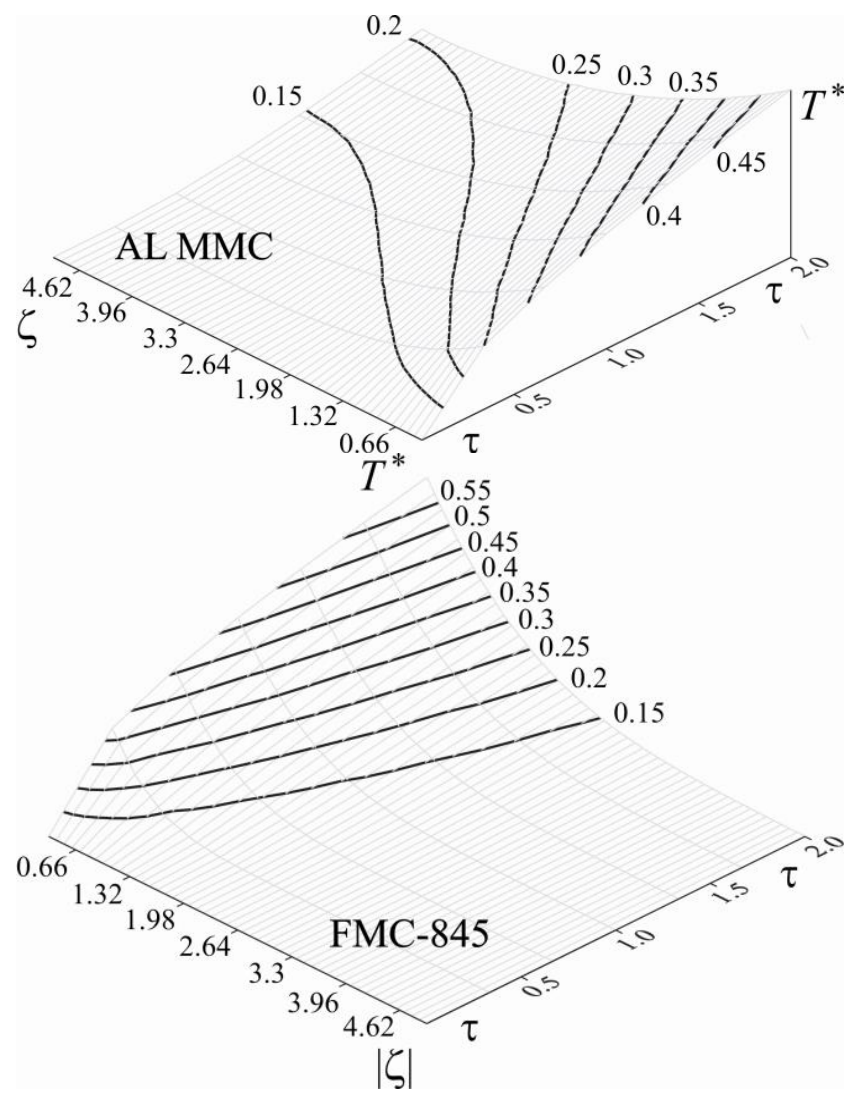

Fig. 4. Spatial and temporal distribution of dimensionless temperature

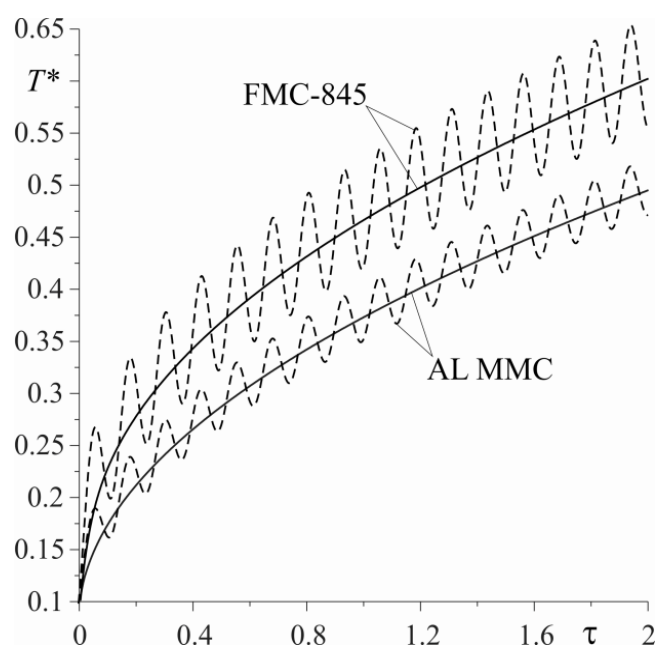

Fig. 5. Evolution of dimensionless temperature $T_{l}^{*}, l=1,2$ on the contact surface $\zeta=0$ at $\omega=0$ (solid lines) and $\omega=50$ (dashed lines)

When using iterative methods to solve nonlinear thermal problems of friction at the initial "zero" step, we must have a solution (preferably the analytical one) to the corresponding linear problems (Yevtushenko et al., 2015). The methodology proposed in this paper proves to be effective when it is difficult or even impossible to obtain such analytical solutions of linear problems. For example there is a change of the specific power of friction in time in the form $q(\tau)=q_{0} q^{*}(\tau)$, when $q^{*}(\tau)=1+\sin (\omega \tau), \tau \geq 0$, where for $\omega=0$ we obtain the problem considered above. Change with time of friction elements temperature at constant $(\omega=0)$ and oscillating $(\omega=50)$ (Kuciej, 2011) specific power of friction is presented in Fig. 5.

\section{CONCLUSIONS}

The solution to one-dimensional nonlinear heat conduction problem of friction for two thermosensitive was obtained by method of lines.

The calculations were conducted for the friction pair (aluminium AL MMC - metal-cermic FMC-845), whose materials are arbitrary nonlinear. Comparison of the results obtained by the method of lines with the results calculated by the method of successive approximations (Och, 2014) was carried out.

Application of the numerical method presented in the article, preceded by the preparation of an appropriate analytical problem (partial linearization), gives perspective to obtain new analytical-numerical solutions to nonlinear heat conduction problems of friction, which cannot be solved by using analytical methods.

Nomenclature: $a$ - characteristic dimension; $b_{i}$ - Biot number; $c$ - specific heat; $c_{0}$-specific heat at an initial temperature; $f$ - friction coefficient; $h$-coefficient of thermal conductivity of contact; $K$ - coefficient of thermal conductivity; $K_{0}$ - coefficient of thermal conductivity at an initial temperature; $k$ - coefficient of thermal diffusivity; $p_{0}$ - pressure; $q_{0}$ - specific power of friction; $T$ - temperature; $T_{0}$ - initial temperature; $T^{*}-$ dimensionless temperature; $t$ - time; $V$ - sliding speed; $z$ - spatial coordinate; $\Theta$ - Kirchhoff's function; $\rho$ - specific density; $\tau$ - Fourier number; $\zeta$ - dimensionless spatial coordinate. 


\section{REFERENCES}

1. Awrejcewicz J., Pyr'yev Yu. (2009), Nonsmooth dynamics of contacting thermoelastic bodies, Springer-Verlag, New York.

2. Barber J. R. (1970), The conduction of heat from sliding solids, Int. J. Heat. Mass Tran., Vol. 13, 857-869.

3. Belyakov N. S., Nosko A. P. (2010), Nonperfect thermal contact of friction bodies, KD LIBROCOM, Moscow, (in Russian).

4. Chichinadze A. V., Braun E. D., Ginsburg A. G., Ignat'eva Z. V. (1979), Calculation, Test and Selection of Frictional Couples, Nauka, Moscow (in Russian).

5. Evtushenko 0, Kuciej M., Och E. (2014 a), Influence of the thermal sensivity of materials on the temperature at friction, Mat. Sci., Vol. 50, No 1, 117-122.

6. Evtushenko O., Kuciej M., Och E. (2014 b), Modeling of temperature conditions for a braking system with regard for the heat sensitivity of materials, Mat. Sci., Vol. 50, No 3, 397-405.

7. Evtushenko 0.0., Pir'ev Yu.O. (1999), Computation of the contact temperature and wear during braking, J. Math. Sci., Vol. 96, 28922896.

8. Gear C. W. (1971), Numerical initial value problems in ordinary differential equations, Prentice-Hall, Englewood Cliffs.

9. Hall G., Watt J. M. (1973), Modern numerical methods for ordinary differential equations, Clarendon Press, Oxford.

10. Kalin M. (2004), Influence of flash temperatures on the tribological behaviour in low-speed sliding: a review, Materials Science and Engineering A, Vol. 374, 390-397.

11. Kirchhoff G. R. (1894), Heat theory lectures, B.G. Teubner, Leipzig (in Germany).

12. Krupowicz A. (1986), Numerical Methods of Initial Value Problems of Ordinary Differential Equations (in Polish), PWN, Warsaw.

13. Kuciej M. (2011), Accounting changes of pressure in time in onedimensional modeling the process of friction heating of disc brake, Int. J. Heat Mass Trans., Vol. 54, 468-474.

14. Kuciej M. (2012), Analytical models sof transient frictional heating, Publisher of Technical University of Bialystok, Bialystok.

15. Kushnir R. M., Popovych V. S. (2011), Heat conduction problems of thermosensitive solids under complex heat exchange. In: Heat conduction - Basic Research, V. Vikhrenko Ed., In Tech, Croatia, 131-154.

16. Nosko A.L., Belyakov N.S., Nosko A.P. (2009), Application of the generalized boundary condition to solving thermal friction problems, J. Frict. Wear, Vol. 30, 615-625.

17. Och E. (2013), Frictional Heating During Sliding of Two Semi-Spaces with Simple Thermal Nonlinearities, Acta Mech. et Autom., Vol. 7, No 4, 236-240.
18. Och E. (2014), Frictional Heating during Sliding of Two Semi-Spaces with Arbitrary Thermal Nonlinearity, Acta Mech. et Autom., Vol. 8, No 4, 204-208.

19. Olesiak Z., Pyryev Yu., Yevtushenko A. (1997), Determination of temperature and wear during braking, Wear, Vol. 210, 120-126.

20. Ozisik M. N. (2000), Finite difference methods in heat transfer, Second Ed., CRC Press, Florida, USA.

21. Podstrigach Ya. S. (1963), The temperature field in a system of rigid bodies coupled by thin interface, Inzh.-Fiz. Zh., Vol. 6, No 10, 129-136, (in Russian).

22. Pyr'yev Yu. (2004), Dynamics of contact systems with respect to heat, friction and wear, Publisher of Technical University of Lodz, Lodz.

23. Rhee S. K., Jacko M. G., Tsang P. H. S. (1991), The role of friction film in friction, wear and noise of automotive brakes, Wear, Vol. 146, No $1,89-97$.

24. Sazonov V. S. (2008), Nonideal contact problem of nonstationary heat conduction for two half-spaces, J. Eng. Phys. Thermophys., Vol. 81, 397-408.

25. Yevtushenko A., Kuciej M., Och E. (2014a), Effect of Thermal Sensitivity of Materials of Tribojoint on Friction Temperature, J. Frict. Wear, Vol. 35, 77-83.

26. Yevtushenko A., Kuciej M., Och E. (2014b), Influence of thermal sensitivity of the pad and disk materials on the temperature during braking, Int. Comm. Heat Mass Transf., Vol. 55, 84-92.

27. Yevtushenko A., Kuciej M., Och E. (2014 c), Temperature in thermally nonlinear pad-disk brake system, Int. Comm. Heat Mass Transf., Vol. 57, 274-281.

28. Yevtushenko A., Kuciej M., Och E. (2015), Some methods for calculating temperature during the friction of thermosensitive materials, Numer. Heat Transf. P. A., V. 67, N 6 (2015), 696-718.

29. Yevtushenko A.A., Kuciej M. (2012), One-dimensional thermal problem of friction during braking: The history of development and actual state, Int. J. Heat Mass Tran., Vol. 55, 4118-4153.

30. Yevtushenko A.A., Kuciej M., Yevtushenko O. (2013), The boundary conditions on the sliding surface in one-dimensional transient heat problem of friction, Int. J. Heat Mass Trans., Vol. 59 , No 1, 1-8.

31. Yune Y.G., Bryant M.D. (1989), Thermal evolution of hot spots in thermally nonlinear carbon graphite sliders, Trans. ASME. J. Tribology, Vol. 111, 591-596.

Acknowledgement. The present paper is financially supported by the National Science Centre of Poland (project No 2011/01/B/ST8/07446). 